\title{
The Safety and Efficacy of Laparoscopic Gastrectomy for Patients with Locally Advanced Gastric Cancer Following Neoadjuvant Chemotherapy
}

\section{Lihang Liu}

Fujian Medical University

\section{Feng Li}

Fujian Provincial Hospital

Shengtao Lin

Fujian Provincial Hospital

Yi Liu

Fujian Medical University

Changshun Yang

Fujian Provincial Hospital

\section{Shaoxin Cai}

Fujian Provincial Hospital

Weihua Li ( $\sim$ liwh2018@sina.com )

Fujian Provincial Hospital https://orcid.org/0000-0002-4752-1693

Research article

Keywords: Laparoscopic surgery, Gastric cancer, Neoadjuvant chemotherapy, Treatment outcome, Survival

Posted Date: July 28th, 2020

DOl: https://doi.org/10.21203/rs.3.rs-47635/v1

License: (a) This work is licensed under a Creative Commons Attribution 4.0 International License. Read Full License 


\section{Abstract}

Background: Limited researches focused on the application of laparoscopic gastrectomy (LG) in locally advanced gastric cancer (LAGC) patients following neoadjuvant chemotherapy (NACT). In this study, we aimed at illustrating the surgical and survival outcome of LG in LAGC patients following NACT.

Methods: We performed a retrospective study of patients with LAGC who received either LG following NACT or upfront LG at Fujian Provincial Hospital between March 2013 and October 2018. Perioperative parameters, short-term and long-term outcomes were compared. The Kaplan-Meier estimator was used to describe the survival curves, and the differences were examined by the log-rank test.

Results: In total, 76 consecutive patients were enrolled into the NACT-LG (41 patients) and LG (35 patients) group, respectively. There was no significant difference between the two groups for baseline characteristics, including age, sex, BMI, Eastern Clinical Oncology Group performance status, tumor size, location, Borrmann type, Lauren type, differentiation, cT stage, and surgical type (all $P>0.05$ ). The surgical trauma in terms of incision length and blood loss, and postoperative recovery in terms of first aerofluxus time, first time on liquid diets, drainage duration, and hospital stays were similar between the two groups (all $P>0.05)$. The operation time was significantly longer for NACT-LG than for LG (286.5 vs. 248.9 min, $\mathrm{P}=0.008)$. There was no significant difference in surgical morbidity $(19.5 \%$ vs. $22.9 \%, \mathrm{P}=0.721)$ between the two groups. No patient died of postoperative complications in the NACT-LG group, and one patient $(1 / 35,2.9 \%)$ died of postoperative complications in the $L G$ group $(P=0.461)$. After NACT, the R0 resection rate was significantly higher $(95.1 \%$ vs. $77.1 \%, P=0.049)$, and metastatic lymph nodes were less for NACTLG than for LG (1 vs. 8, $P=0.001)$. Compared with the LG group, the NACT-LG group had a significantly better DFS (59.4\% vs. $14.4 \%, \mathrm{P}=0.034)$ and better OS $(69.0 \%$ vs. $37.4 \%, \mathrm{P}=0.009)$ at 3 years.

Conclusions: NACT does not decrease safety of LG for patients with LAGC and offer higher R0 resection rate and better disease-free and overall survival. For patients with LAGC, LG following NACT should be the priority treatment.

\section{Background}

Gastric cancer is a life-threatening disease, and surgical resection remains the only curative treatment [1, 2]. However, the long-term outcome is still far from satisfactory for patients who receive surgery alone, especially in patients with locally advanced gastric cancer (LAGC). Approximately $70 \%$ patients with LAGC died within 5 years after surgery $[3,4]$. Therefore, perioperative therapy is imperatively required to improve the survival.

Neoadjuvant chemotherapy (NACT) is generally accepted to benefit prognosis by downstaging tumor, increasing complete resection rate and eradicating micro-metastases [5, 6]. The MAGIC study has illustrated a survival benefit of perioperative chemotherapy and consequently opened the era of neoadjuvant therapy in patients with LAGC [7]. Although NACT has demonstrated several strengths as mentioned above, surgeons concerned about its negative effects on the surgical safety. Destruction of 
anatomical dissection plane induced by tissue edema and fibrotic changes may complicate laparoscopic surgeries. In addition, chemotherapy related adverse effects deteriorate the nutritional and immune status of patients, which may impair the prognosis of patients [8-10].

With the development of laparoscopic technique and accumulation of evidence from clinical trials in recent years, laparoscopic surgery has been recommended to patients with LAGC [11]. Nevertheless, limited studies focused on the application of laparoscopic gastrectomy (LG) in patients receiving NACT. There is an urgent need for researches comparing patients receiving laparoscopic surgery after NACT or upfront laparoscopic surgery. However, conducting prospective trials targeting on the issue is impractical for the reason that NACT has been widely accepted in patients with locally advanced gastric cancer (LAGC) due to its positive effects on patients' survival [12-14].

Therefore, this retrospective study was conducted to evaluate the safety and efficacy of LG for patients with LAGC following NACT, focusing on whether NACT decreased the safety of LG for patients with LAGC.

\section{Methods}

\section{Patients}

We reviewed our prospectively maintained gastric cancer database at Fujian Provincial Hospital. Clinical data of LAGC patients underwent either LG following NACT or upfront LG between March 2013 and October 2018 were analyzed. The inclusion criteria were listed as follows: (1) stomach adenocarcinoma, histologically confirmed by endoscopic biopsy; (2) clinical stage $\otimes$ (cT3/4a, N+, M0) according to the 8th edition of the AJCC/UICC staging system [15], diagnosed using computed tomography (CT), endoscopic ultrasonography (EUS), or laparoscopic exploration. The exclusion criteria were listed as follows: (1) cancer of the esophagogastric junction; (2) residual gastric cancer; (3) malignant tumor history; and (4) emergency surgery due to complications (obstruction, bleeding, or perforation). All patients signed written informed consent, and this study was approved by the ethics committee of the Fujian Provincial Hospital.

\section{Treatment}

Patients in the NACT-LG group received three to six cycles of NACT (XELOX regimen) at three-weekly intervals. Surgery was performed four to five weeks after the last cycle. Patients in the LG group received upfront surgery after preoperative evaluation. Laparoscopic total or distal gastrectomy with D2 lymphadenectomy was performed for patients according to the location of tumor. Reconstruction of the gastrointestinal tract was all completed using Roux-en-Y anastomosis. All the operations were performed by one experienced surgeon team. Within five weeks after surgery, patients were treated with three to six cycles of postoperative chemotherapy (XELOX regimen) at three-weekly intervals.

\section{Clinical and pathologic assessment}

Clinicopathological variables, such as age, sex, body mass index (BMI), Eastern Clinical Oncology Group performance status (ECOG PS) [16], tumor size, tumor location, Borrmann type, Lauren type, cT stage, as 
well as perioperative data, such as surgical type, incision length, operation time, blood loss, first aerofluxus time, drainage duration, postoperative hospital stay, and surgical radicalness were analyzed. Clavien-Dindo classification system was applied to evaluate postoperative complication $[17,18]$. Postoperative mortality were defined as death of any cause within 30 days after surgery. Pathological findings including number of harvested and metastatic lymph nodes, pathological stage, and tumor regression grade (TRG) were also compared between groups. The TRG was evaluated according to the NCCN guideline [12].

\section{Follow-up}

All the patients received postoperative follow-up every 3 months within 2 years after surgery and every 6 months for the next 3 years. Recurrence was defined as local recurrence identified by contrast CT scan or endoscopy biopsy, or distant recurrence identified by CT scan, ECT bone scan or PET/CT. Immediate follow-up occurred in patients with worsening or new symptoms. The disease-free survival (DFS) was calculated from surgery to recurrence, death, or last follow-up; the overall survival (OS) was calculated from beginning of treatment to death or last follow-up.

\section{Statistical analysis}

Continuous variables with normal distribution were expressed as the mean \pm standard deviation (SD) while non-normal variables were expressed as the median (range). T-test or Mann-Whitney rank-sum test were used to evaluate differences between the two groups according to the distribution of data. The Kaplan-Meier method was performed using a log-rank test to estimate differences in DFS and OS. SPSS version 20 was used for the statistical analysis, and $P$ values less than 0.05 (two-sided) were considered statistically significant.

\section{Results}

\section{Patients}

There were 76 patients in this study, 41 (53.9\%) patients in the NACT-LG group and $35(46.1 \%)$ patients in the LG group, respectively. The characteristics of patients were detailed in Table 1. The two groups were balanced in baseline characteristics in term of age, sex, BMI and ECOG PS (all $P>0.05$ ). There were also no differences between groups in tumor size, tumor location, Borrmann type, Lauren type, differentiation and cT stage (all $P>0.05$ ). 
Table 1

Patient baseline characteristics in the NACT-LG and LG groups

\begin{tabular}{|c|c|c|c|}
\hline & $\begin{array}{l}\text { NACT-LG } \\
(\mathrm{n}=41)\end{array}$ & $\begin{array}{l}\text { LG } \\
(n=35)\end{array}$ & $\mathbf{P}$ \\
\hline Age (y) & & & 0.112 \\
\hline$<60$ & $19(46.3)$ & $10(28.6)$ & \\
\hline$\geq 60$ & $22(53.7)$ & 25 (71.4) & \\
\hline Sex & & & 0.231 \\
\hline Male & $32(78.0)$ & $23(65.7)$ & \\
\hline Female & $9(22.0)$ & $12(34.3)$ & \\
\hline BMI $\left(\mathrm{kg} / \mathrm{m}^{2}\right)$ & $23.2 \pm 1.9$ & $22.6 \pm 2.8$ & 0.238 \\
\hline ECOG PS & & & 0.760 \\
\hline 0 & $26(63.4)$ & $21(60.0)$ & \\
\hline 1 & $15(36.6)$ & $14(40.0)$ & \\
\hline Tumor size $(\mathrm{cm})$ & & & 0.577 \\
\hline$<5$ & $26(63.4)$ & $20(57.1)$ & \\
\hline$\geq 5$ & 15 (36.6) & $15(42.9)$ & \\
\hline Tumor location & & & 0.187 \\
\hline Upper third & 15 (36.6) & $6(17.1)$ & \\
\hline Middle third & $14(34.1)$ & $14(40.0)$ & \\
\hline Lower third & $12(29.3)$ & $14(40.0)$ & \\
\hline Total & $0(0)$ & $1(2.9)$ & \\
\hline Borrmann type & & & 0.117 \\
\hline प & $1(2.4)$ & $2(5.7)$ & \\
\hline ૫ & $7(17.2)$ & $1(2.9)$ & \\
\hline ( & $32(78.0)$ & $29(82.9)$ & \\
\hline प & $1(2.4)$ & $3(8.6)$ & \\
\hline \multicolumn{3}{|l|}{ Lauren type } & 0.913 \\
\hline \multicolumn{4}{|c|}{$\begin{array}{l}\text { NACT, neoadjuvant chemotherapy; LG, laparascopic gastrectomy; BMI, body mass index; ECOG PS, } \\
\text { Eastern Clinical Oncology Group performance status; Fisher's exact test was used as an alternative to } \\
\text { Chi-square test when the number in one of the cells is smaller than } 5 \text {. }\end{array}$} \\
\hline
\end{tabular}




\begin{tabular}{|llll|}
\hline & $\begin{array}{l}\text { NACT-LG } \\
(\mathbf{n = 4 1 )}\end{array}$ & $\begin{array}{l}\text { LG } \\
(\mathbf{n}=\mathbf{3 5})\end{array}$ \\
\hline Diffused & $9(22.0)$ & $9(25.7)$ & P \\
\hline Intestinal & $18(43.9)$ & $14(40.0)$ & 0.367 \\
\hline Mixed & $14(34.1)$ & $12(34.3)$ & \\
\hline cT stage & & $19(54.3)$ \\
\hline T3 & $18(43.9)$ & $16(45.7)$ \\
\hline T4 & $23(56.1)$ & \\
\hline $\begin{array}{l}\text { NACT, neoadjuvant chemotherapy; LG, laparascopic gastrectomy; BMI, body mass index; ECOG PS, } \\
\text { Eastern Clinical Oncology Group performance status; Fisher's exact test was used as an alternative to } \\
\text { Chi-square test when the number in one of the cells is smaller than 5. }\end{array}$ \\
\hline
\end{tabular}

\section{Surgical procedures}

All patients received an LG with D2 lymphadenectomy. The surgical data were compared in Table 2. No significant difference was found in the surgical trauma in terms of incision length and blood loss, and postoperative recovery in terms of first aerofluxus time, and first time on liquid diets between the two groups (all $P>0.05$ ). Patients in $L G$ group tended to have shorter drainage duration ( $8 v s .7 d, P=0.061$ ) and shorter postoperative hospital stay ( $10 \mathrm{vs} .9 \mathrm{~d}, \mathrm{P}=0.059)$, however no significant difference was observed. Despite having a longer operation time (286.5 vs. $248.9 \mathrm{~min}, \mathrm{P}=0.008)$, the NACT-LG exhibited a significantly greater $\mathrm{R} 0$ resection rate $(95.1 \%$ vs. $77.1 \%, \mathrm{P}<0.05)$. $\mathrm{R} 1$ resection was performed in two (4.9\%) patients in the NACT-LG group with positive proximal margins and eight (22.9\%) patients in the LG group, including four (11.4\%) patients with positive proximal margins and four (11.4\%) patients with positive distal margins. 
Table 2

Comparison of surgical procedures between the NACT-LG and LG groups

\begin{tabular}{|c|c|c|c|}
\hline & NACT-LG $(n=41)$ & $L G(n=35)$ & $\mathbf{P}$ \\
\hline Surgical type & & & 0.327 \\
\hline LTG & $30(73.2)$ & $21(60.0)$ & \\
\hline LDG & $11(26.8)$ & $14(40.0)$ & \\
\hline Incision length (cm) & $6.0(3.0-8.0)$ & $5.0(4.0-7.0)$ & 0.109 \\
\hline Operation time (min) & $286.5(205.0-470.0)$ & $248.9(140.0-360.0)$ & 0.008 \\
\hline Estimated blood loss (mL) & $100.0(20.0-450.0)$ & $100.0(30.0-2500.0)$ & 0.395 \\
\hline The first aerofluxus time (days) & $3.0(1.0-6.0)$ & $3.0(1.0-6.0)$ & 0.303 \\
\hline $\begin{array}{l}\text { Time to pull drainage } \\
\text { (days) }\end{array}$ & $8.0(5.0-13.0)$ & $7.0(5.0-14.0)$ & 0.061 \\
\hline First time on liquid diets (days) & $3.0(2.0-12.0)$ & $3.0(1.0-12.0)$ & 0.118 \\
\hline Hospital stay after surgery (days) & $10.0(6.0-20.0)$ & $9.0(6.0-21.0)$ & 0.059 \\
\hline Surgical radicalness & & & 0.049 \\
\hline Ro & $39(95.1)$ & $27(77.1)$ & \\
\hline R1 & $2(4.9)$ & $8(22.9)$ & \\
\hline
\end{tabular}

\section{Surgery morbidity and mortality}

The postoperative complications were detailed in Table 3. Postoperative complications occurred similarly in two groups, 8 patients $(19.5 \%)$ in the NACT-LG group and 8 patients $(22.9 \%)$ in the LG group $(\mathrm{P}=$ 0.721). The most common postoperative complications were intra-abdominal infection $(7 / 76,9.2 \%)$, followed by abdominal bleeding $(6 / 76,7.9 \%)$ and pulmonary infection $(5 / 76,6.6 \%)$, respectively. Three (8.6\%) patients suffered major complications (grade III- $\mathrm{X}$ ) requiring invasive interventions in the LG group; one $(2.9 \%)$ patient with anastomotic leakage recovered after active abodominal drainage; one $(2.9 \%)$ patient with heart failure recovered after ICU care, and one (2.9\%) patient died of respiratory failure. No patient died of postoperative complications in the NACT-LG group $(P=0.461)$. 
Table 3

Postoperative complications in the NACT-LG and LG groups

\begin{tabular}{|c|c|c|c|}
\hline & NACT-LG $(n=41)$ & LG $(n=35)$ & $\mathbf{P}$ \\
\hline Grade I & $0(0)$ & $0(0)$ & NA \\
\hline 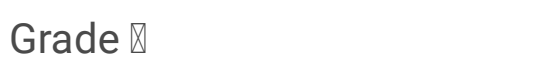 & 8 (19.5) & $5(14.3)$ & 0.546 \\
\hline Transfusion & $4(9.8)$ & $2(5.7)$ & 0.822 \\
\hline Pulmonary infection & $2(4.9)$ & $3(8.6)$ & 0.855 \\
\hline Intra-abdominal infection & $4(9.8)$ & $3(8.6)$ & $>0.05$ \\
\hline Intra-abdominal hemorrhage & $1(2.4)$ & $0(0)$ & $>0.05$ \\
\hline Grade III & $0(0)$ & $1(2.9)$ & 0.461 \\
\hline Anastomotic leakage & $0(0)$ & $1(2.9)$ & \\
\hline Grade $\otimes$ & $0(0)$ & $1(2.9)$ & 0.461 \\
\hline Heart failure & $0(0)$ & $1(2.9)$ & \\
\hline Grade $\otimes$ & $0(0)$ & $1(2.9)$ & 0.461 \\
\hline Respiratory failure & $0(0)$ & $1(2.9)$ & \\
\hline Surgical morbidity & $8(19.5)$ & $8(22.9)$ & 0.721 \\
\hline Surgical mortality & $0(0)$ & $1(2.9)$ & 0.461 \\
\hline
\end{tabular}

\section{Pathological outcomes}

A comparison of pathologies between the two groups was summarized in Table 4. The number of resected lymph nodes did not differ significantly between the two groups, whereas significantly less metastatic lymph nodes were found in the NACT-LG group than in the LG group ( $1 \mathrm{vs} .8, \mathrm{P}=0.001)$. Among all patients, there was a greater proportion of less advanced $\mathrm{pT}$ stage $(\mathrm{T} 0-2 ; 29.3 \%$ vs. $0 \%, \mathrm{P}<$

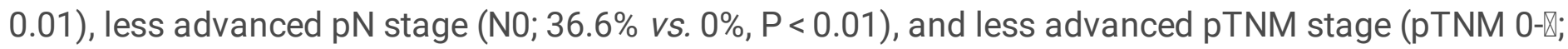
$51.2 \%$ vs. $0 \%, P<0.01$ ) in the NACT-LG group than in the LG group. In the NACT-LG group, the pathological response was $65.9 \%$, and the samples of each TRG were listed in Fig. 1. Over half of the patients (51.2\%) obtained tumor downstaging in the NACT-LG group. Four patients $(9.8 \%)$ were considered pathological complete responders after preoperative chemotherapy. 
Table 4

Comparison of pathologies between the NACT-LG and LG groups

\begin{tabular}{|c|c|c|c|}
\hline & NACT-LG $(n=41)$ & LG $(n=35)$ & $\mathbf{P}$ \\
\hline Number of resected lymph nodes & $37(12-91)$ & $32(12-69)$ & 0.081 \\
\hline Number of metastatic lymph nodes & $1(0-36)$ & $8(1-27)$ & 0.001 \\
\hline pT stage & & & 0.008 \\
\hline $0-2$ & $12(29.3)$ & $0(0)$ & \\
\hline $3-4$ & $29(70.7)$ & $35(100.0)$ & \\
\hline pN stage & & & $<0.01$ \\
\hline 0 & $15(36.6)$ & $0(0)$ & \\
\hline $1-3$ & $26(63.4)$ & $35(100.0)$ & \\
\hline pTNM stage & & & $<0.01$ \\
\hline $0-\rrbracket$ & $21(51.2)$ & $0(0)$ & \\
\hline Q & $20(48.8)$ & $35(100.0)$ & \\
\hline Tumor regression grades & & & NA \\
\hline 0 & $4(9.8)$ & NA & \\
\hline 1 & $13(31.7)$ & NA & \\
\hline 2 & $10(24.4)$ & NA & \\
\hline 3 & $14(34.1)$ & NA & \\
\hline Pathological complete response & $4(9.8)$ & NA & \\
\hline \multicolumn{4}{|c|}{$\begin{array}{l}\text { NACT, neoadjuvant chemotherapy; LG, laparascopic gastrectomy; NA, not applicable; Fisher's exact } \\
\text { test was used as an alternative to Chi-square test when the number in one of the cells is smaller than } \\
5 .\end{array}$} \\
\hline
\end{tabular}

\section{Survival}

The overall median follow-up was 23.0 months (6.6-71.5) in this study, 25.6 months (9.5-71.5) in the NACT-LG group, and 18.6 months (6.6-40.6) in the LG group, respectively. The survival curves were shown in Fig. 2. The 3-year disease-free survival rates in the NACT-LG group and LG group were 59.4\% and $14.4 \%(P=0.034)$, respectively. The 3-year overall survival rates in the NACT-LG group and LG group were $69.0 \%$ and $37.4 \%(P=0.009)$, respectively.

\section{Discussion}


LAGC is characterized by low radical resection rate, high relapse rate and high mortality. Laparoscopic surgery combined with perioperative therapy has been widely accepted as the mainstream treatment. However, only few studies focused on the safety and efficacy of laparoscopic surgery in patients after NACT. As far as we known, there are only few studies comparing the surgical and survival outcome between upfront LG and LG after NACT. The present study revealed that NACT did not decrease safety of LG for patients with LAGC and might offer survival benefits compared with upfront LG.

We found that LG following NACT did not increase the severity of surgical trauma, or delay postoperative recovery. Although operation time was extended for NACT-LG than for LG, the postoperative complications were comparable, which was in consistent with a previous study reported by An et al [8]. It was reported that the negative effects of chemotherapy on operation might be overcome by laparoscope. Laparoscopic surgery offers amplifying visual, better exposure of anatomical hierarchy, and then contributes to delicate anatomy of blood vessels and lymphatic vessels [19]. On the other hand, adverse effects caused by chemotherapy can be attenuated by optimized perioperative care. For instance, malnutrition caused by gastrointestinal side effects can be treated by adequate perioperative nutrition.

Micro-metastases outside the surgical region and microscopic positive margin were the main causes of treatment failure in LAGC [20-22]. NACT may benefit these patients by eradicating metastases and potentially downstaging tumor, thereby increasing the R0 resection rate. Among 41 patients receiving NACT in this study, the pathological response was $65.9 \%$, in concordance with previous studies $[23,24]$. Over half of patients achieved tumor downstaging, which seemed attributable to the effects of NACT. Moreover, our study demonstrated the benefits of LG following NACT compared with immediate surgery, with an increase of 18 percent in the $\mathrm{R} 0$ resection rate $(95.1 \% \mathrm{vs} .77 .1 \%, \mathrm{P}=0.049)$ and a reduction in the number of metastatic lymph nodes $(1 \mathrm{vs} .8, \mathrm{P}=0.001)$.

The MAGIC trial and the EORTC study disagreed about the survival benefits of perioperative chemotherapy in resectable gastric cancer patients $[7,25]$. Since proportions of D2 dissection were different among two studies (MAGIC: 42.5\%; EORTC: 95.7\%), whether patients with D2 gastrectomy benefited from NACT remained unclear. The present study, with all patients received a D2 lymph node dissection, showed that 3-year DFS and OS were superior for NACT-LG than LG (DFS: $59.4 \%$ vs. $14.4 \%$, OS: $69.4 \%$ vs. $37.4 \%)$. We speculated that sufficient preoperative chemotherapy courses and better compliance to chemotherapy may enhance the positive effects of NACT on survival. However, multicenter, large-sample clinical trials were required to verify this hypothesis in the future.

Despite positive findings mentioned above, there were several limitations in this study. First, as a retrospective study with non-randomized patient groups, selection bias were inevitable in this study. Second, the generalizability of findings drawn from a single-center study may be limited. Third, the followup period was too short to analyze the longer survival situation.

\section{Conclusions}


In summary, for patients with LAGC who underwent $L G$, this study provided supportive evidence favoring the application of NACT. NACT does not decrease safety of LG for patients with LAGC and offer higher R0 resection rate and better disease-free and overall survival. For patients with LAGC, LG following NACT should be the priority treatment.

\section{Abbreviations}

BMI, body mass index; DFS, disease-free survival; ECOG PS, Eastern Clinical Oncology Group performance status; LAGC, locally advanced gastric cancer; LDG, laparoscopic distal gastrectomy; LG, laparascopic gastrectomy; LTG, laparoscopic total gastrectomy; NA, Not applicable; NACT, neoadjuvant chemotherapy; OS, overall survival; TRG, tumor regression grade.

\section{Declarations}

\section{Acknowledgments}

Not applicable

\section{Authors' contributions}

LHL, FL and WHL designed the study. LHL, FL, STL, YL, CSY, SXC acquired the clinical data. LHL and FL performed the statistical analysis. The draft manuscript was prepared by LHL and FL. WHL provided critical revision of drafts. All authors read and approved the final manuscript.

\section{Funding}

This work was not funded by any grant.

\section{Availability of data and materials}

The datasets used and/or analysed during the current study available from the corresponding author on reasonable request.

\section{Ethics approval and consent to participate}

This study was approved by the ethics committee of the Fujian Provincial Hospital. All patients signed written informed consent.

\section{Consent for publication}




\section{Competing interests}

The authors declare that they have no competing interests.

\section{References}

1. Lutz MP, Zalcberg JR, Ducreux M, Ajani JA, Allum W, Aust D, et al. Highlights of the EORTC St. Gallen International Expert Consensus on the primary therapy of gastric, gastroesophageal and oesophageal cancer - Differential treatment strategies for subtypes of early gastroesophageal cancer. Eur J Cancer. 2012;48:2941-53.

2. Van Cutsem E, Dicato M, Geva R, Arber N, Bang Y, Benson A, et al. The diagnosis and management of gastric cancer: expert discussion and recommendations from the 12th ESMO/World Congress on Gastrointestinal Cancer, Barcelona, 2010. Ann Oncol. 2011;22:v1-9.

3. Sant M, Allemani C, Santaquilani M, Knijn A, Marchesi F, Capocaccia R. EUROCARE-4. Survival of cancer patients diagnosed in 1995-1999. Results and commentary. Eur J Cancer. 2009;45:931-91.

4. Ajani JA, Bentrem DJ, Besh S, D’Amico TA, Das P, Denlinger C, et al. Gastric Cancer, Version 2.2013: featured updates to the NCCN Guidelines. J Natl Compr Canc Netw. 2013;11:531-46.

5. Song Z, Wu Y, Yang J, Yang D, Fang X. Progress in the treatment of advanced gastric cancer. Tumor Biol. 2017;39:1010428317714626.

6. Miao Z-F, Liu X-Y, Wang Z-N, Zhao T-T, Xu Y-Y, Song Y-X, et al. Effect of neoadjuvant chemotherapy in patients with gastric cancer: a PRISMA-compliant systematic review and meta-analysis. BMC Cancer. 2018;18:118.

7. Cunningham D, Allum WH, Stenning SP, Thompson JN, Van de Velde CJH, Nicolson M, et al. Perioperative chemotherapy versus surgery alone for resectable gastroesophageal cancer. N Engl J Med. 2006;355:11-20.

8. An JY, Kim KM, Kim YM, Cheong J-H, Hyung WJ, Noh SH. Surgical Complications in Gastric Cancer Patients Preoperatively Treated with Chemotherapy: Their Risk Factors and Clinical Relevance. Ann Surg Oncol. 2012;19:2452-8.

9. Awad S, Tan BH, Cui H, Bhalla A, Fearon KCH, Parsons SL, et al. Marked changes in body composition following neoadjuvant chemotherapy for oesophagogastric cancer. Clin Nutr. 2012;31:74-7.

10. Tegels JJ. Improving the outcomes in gastric cancer surgery. World J Gastroenterol. 2014;20:13692704.

11. Yu J, Huang C, Sun Y, Su X, Cao H, Hu J, et al. Effect of Laparoscopic vs Open Distal Gastrectomy on 3-Year Disease-Free Survival in Patients With Locally Advanced Gastric Cancer: The CLASS-01 Randomized Clinical Trial. JAMA. 2019;321:1983-92. 
12. National Comprehensive Cancer Network. NCCN clinical practice guidelines in oncology (NCCN guidelines): gastric cancer. (Version 2. 2019). 2019.

https://www.nccn.org/professionals/physician_gls/pdf/gastric.pdf.

13. Japanese Gastric Cancer Association. Japanese gastric cancer treatment guidelines 2018 (5th edition). Gastric Cancer. 2020;20:1-21.

14. Wang F-H, Shen L, Li J, Zhou Z-W, Liang H, Zhang X-T, et al. The Chinese Society of Clinical Oncology (CSCO): clinical guidelines for the diagnosis and treatment of gastric cancer. Cancer Commun Lond Engl. 2019;39:10.

15. Amin MB, Edge SB, Greene FL, Brierley JD. AJCC cancer staging manual. 8th ed. New York: Springer; 2017.

16. Oken MM, Creech RH, Tormey DC, Horton J, Davis TE, McFadden ET, et al. Toxicity and response criteria of the Eastern Cooperative Oncology Group. Am J Clin Oncol. 1982;5:649-55.

17. Clavien PA, Sanabria JR, Strasberg SM. Proposed classification of complications of surgery with examples of utility in cholecystectomy. Surgery. 1992;111:518-26.

18. Dindo D, Demartines N, Clavien P-A. Classification of surgical complications: a new proposal with evaluation in a cohort of 6336 patients and results of a survey. Ann Surg. 2004;240:205-13.

19. Li Z, Shan F, Ying X, Zhang Y, E J-Y, Wang Y, et al. Assessment of Laparoscopic Distal Gastrectomy After Neoadjuvant Chemotherapy for Locally Advanced Gastric Cancer: A Randomized Clinical Trial. JAMA Surg. 2019;154:1093-101.

20. Wang S-Y, Yeh C-N, Lee H-L, Liu Y-Y, Chao T-C, Hwang T-L, et al. Clinical Impact of Positive Surgical Margin Status on Gastric Cancer Patients Undergoing Gastrectomy. Ann Surg Oncol. 2009;16:273843.

21. Bickenbach KA, Gonen M, Strong V, Brennan MF, Coit DG. Association of Positive Transection Margins with Gastric Cancer Survival and Local Recurrence. Ann Surg Oncol. 2013;20:2663-8.

22. Nagata T, Ichikawa D, Komatsu S, Inoue K, Shiozaki A, Fujiwara H, et al. Prognostic impact of microscopic positive margin in gastric cancer patients. J Surg Oncol. 2011;104:592-7.

23. Tanaka Y, Kunisaki C, Izumisawa Y, Makino H, Kimura J, Sato S, et al. A Phase I/II Study of NAC with Docetaxel, Cisplatin, and S-1 for Stage III Gastric Cancer. Anticancer Res. 2018;38:6015-21.

24. Shinkai M, Imano M, Chiba Y, Iwama M, Shiraisi O, Yasuda A, et al. Phase II trial of neoadjuvant chemotherapy with intraperitoneal paclitaxel, S-1, and intravenous cisplatin and paclitaxel for stage IIIA or IIIB gastric cancer. J Surg Oncol. 2019;119:56-63.

25. Schuhmacher C, Gretschel S, Lordick F, Reichardt P, Hohenberger W, Eisenberger CF, et al. Neoadjuvant chemotherapy compared with surgery alone for locally advanced cancer of the stomach and cardia: European Organisation for Research and Treatment of Cancer randomized trial 40954. J Clin Oncol. 2010;28:5210-8.

\section{Figures}




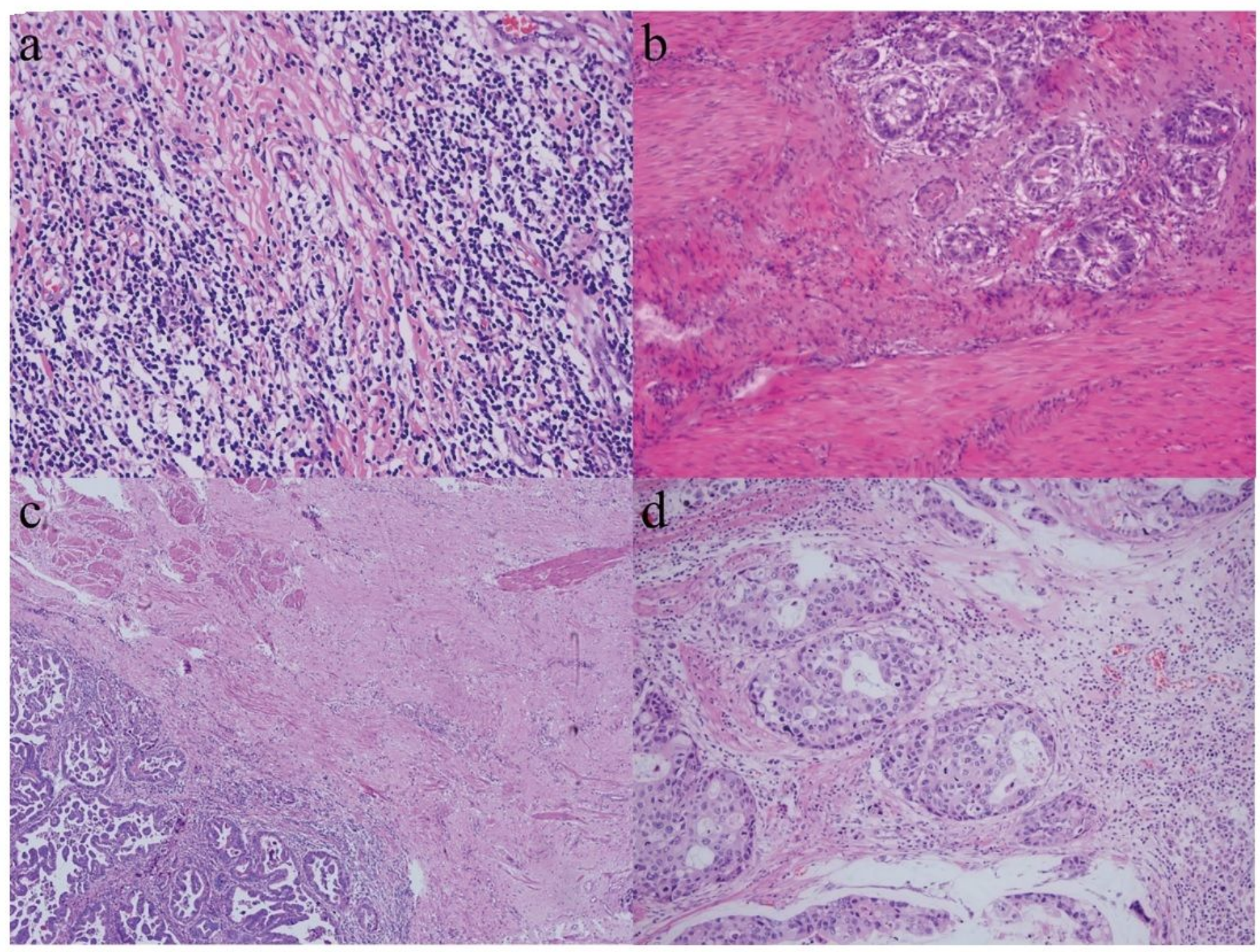

Figure 1

The samples of each TRG of patients in the NACT- LG group. a TRG 0 after H-E staining $(\times 20)$. b TRG 1 after H-E staining $(\times 10)$. c TRG 2 after H-E staining $(\times 4)$. d TRG 3 after H-E staining $(\times 20)$. TRG, tumor regression grade; NACT, neoadjuvant chemotherapy; $L G$, laparascopic gastrectomy. 
a

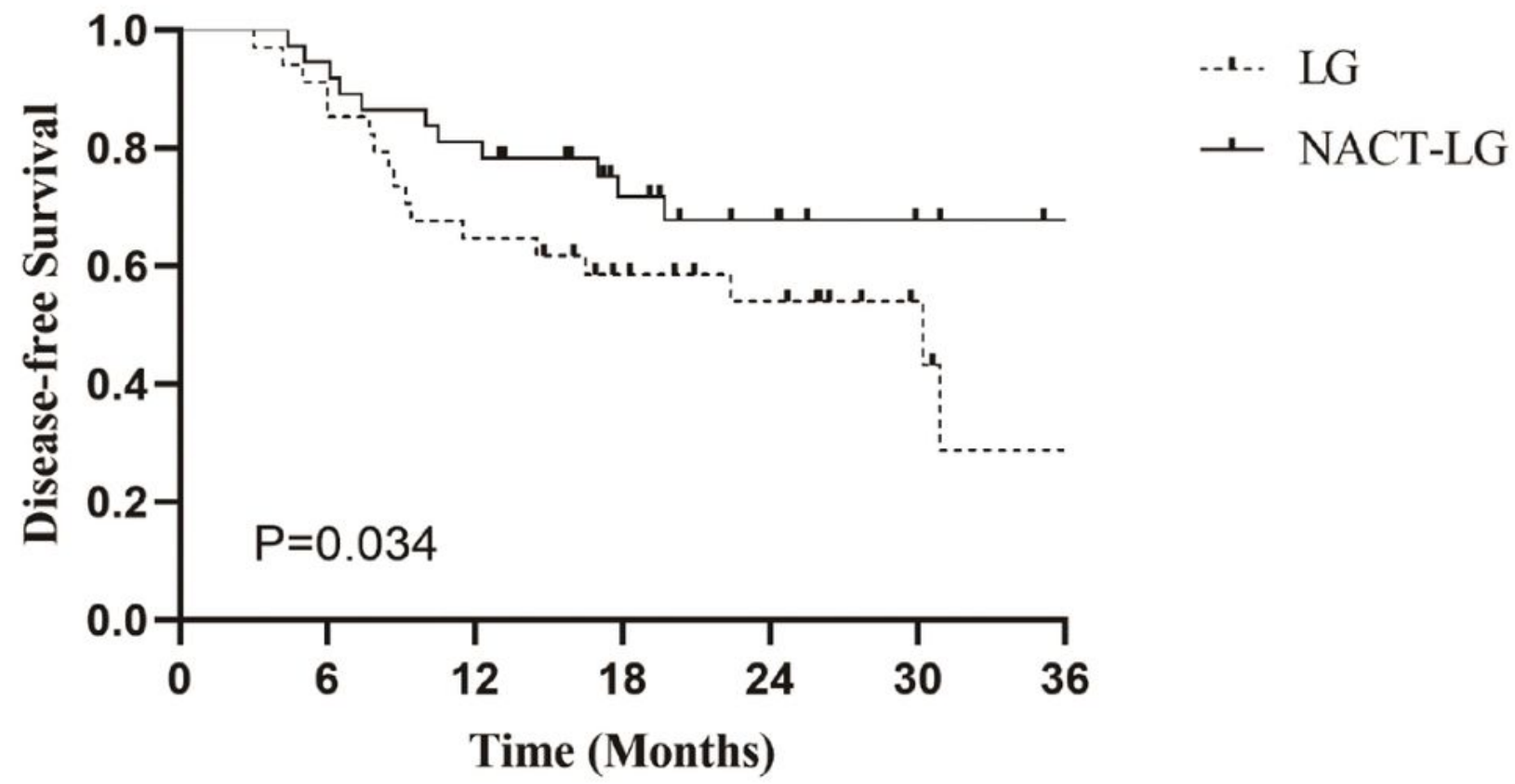

b

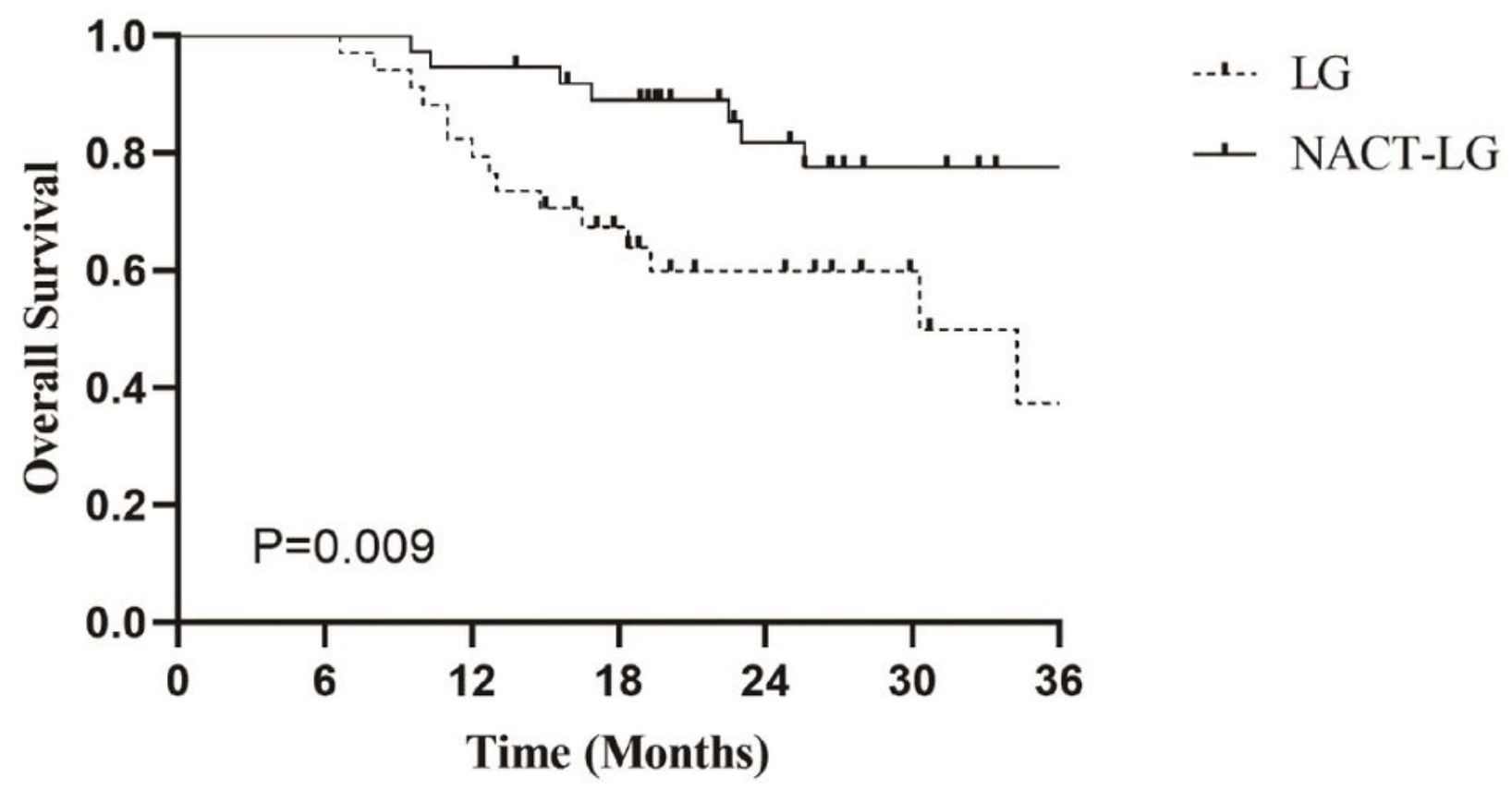

Figure 2

Kaplan-Meier estimates of a disease-free survival and b overall survival. 